\title{
Population status and nest success of the Critically Endangered Mariana Crow Corvus kubaryi on Rota, Northern Mariana Islands
}

\author{
LAINIE ZARONES, ADRIENNE SUSSMAN, JOHN M. MORTON, \\ SHELDON PLENTOVICH, SARAH FAEGRE, CELESTINO AGUON, \\ ARJUN AMAR and RENEE ROBINETTE HA
}

\section{Summary}

The Mariana Crow Corvus kubaryi is a Critically Endangered species found only on the island of Rota, Northern Mariana Islands. It was extirpated from the neighbouring island of Guam by the introduced brown tree snake Boiga irregularis and the Rota population has been in decline since at least 1995 . We identified only 6o pairs present on Rota in 2007 compared with an estimated 117 pairs in 1998 , a decline of nearly $50 \%$ in nine years. The decline may be linked to proximity to human activities, though more data are needed. We monitored 204 crow nests between the 1996 and 2009 breeding seasons. Crows initiate clutches between August and April. The overall estimate of nest success was $25.7 \%(n=204)$. On average $49 \%$ of pairs produced at least one fledgling per season. The mean number of fledglings per pair per year is 0.66 . Mean clutch size was $2.6(n=82)$, mean number of nestlings was $1.4(n=106)$, and mean number of fledglings per nest was $1.3(n=68)$. Daily survival rates declined in later years, and increased during the nest cycle. The number of pairs with successful nests did not change during the study period, nor did the number of fledglings per pair. Predation appeared to be the primary cause of nest failure. The breeding season lasted around nine months and pairs re-nested after failure. Predation of adults and juveniles by cats, combined with possible inbreeding depression, habitat disturbance and human persecution appear to be the cause of decline of the Mariana Crow. We strongly recommend a programme of invasive predator control, habitat maintenance, and captive rearing to ensure the species' survival.

\section{Introduction}

Island species are especially vulnerable to extinction, due to a combination of limited geographic range, small population sizes, and low resistance to introduced predators and pathogens (Steadman 2006). These risks are particularly high for avifauna and as a result more than $90 \%$ of all bird extinctions recorded in the past 400 years have been of island species (Newton 1998). The threats to birds can be expected to accelerate as increasing human development of islands leads to further habitat alteration.

The Marianas archipelago is one such critical location, where some populations of forest birds have dropped precipitously during the twentieth century. The avifauna on Guam, the southernmost island in the archipelago, was decimated by the introduction of the brown tree snake Boiga irregularis after World War II (Savidge 1987). All but one forest bird species on the neighbouring island of Rota have also suffered significant declines in number and range within the past 20 years (Amar et al. 2008). In contrast, some of the same species on the neighbouring islands of Saipan 
and Tinian are stable or have increased in recent years (Camp et al. 2009, 2012), suggesting that the factors causing population declines on Rota are reduced or absent on Saipan and Tinian (Amar et al. 2008).

One of the most critically affected avian species in the Mariana Islands is the Mariana Crow or Aga Corvus kubaryi, which is endemic to Guam and Rota. The Mariana Crow is listed as federally endangered (USFWS 1984) and as 'Critically Endangered' by IUCN (2011). The species was considered common on Guam until the 1960s (Jenkins 1983), but had declined to an estimated 100 individuals by 1985 (Michael 1987) due to predation of Mariana Crow nests by the brown tree snake (Savidge 1987, Wiles et al. 2003). Despite efforts to use electrical barriers to protect nests from snakes (Aguon et al. 2002), the crow was extirpated from Guam by the late 1990s (USFWS 2005).

Although the brown tree snake is not established on Rota (Rodda and Savidge 2007), the Rota population of Mariana Crows has also declined dramatically in recent decades. An island-wide survey on Rota in 1982 suggested a Mariana Crow population of 1,350 individuals (Engbring et al. 1986). By 1995, the population was estimated at 590 individuals, a decline of $56 \%$ in 13 years (Fancy et al. 1999). Detections of Mariana Crows at off-road survey stations declined $83 \%$ from 1982 to 1998 , a trend that was confirmed by the $67 \%$ decline in population estimates using variable circular plot methodology during this same time period (Plentovich et al. 2005). A survey of territorial Mariana Crow pairs during 1996-1999 estimated that only 117 breeding pairs remained on Rota (Plentovich et al. 2005). Point count surveys on Rota showed a $93 \%$ decline in the Mariana Crow population from 1982 to 2004 - the largest population decline of all forest birds on Rota during this time (Amar et al. 2008).

In contrast to the clear effects of snake predation of Mariana Crow nests on Guam, a range of natural and anthropogenic factors have been suggested as possible causes of the decline of the Mariana Crow on Rota. These include habitat loss and degradation, persecution by humans, predation by introduced cats Felis catus and monitor lizards Varanus indicus, predation and competition by rats Rattus diardii, R. exulans and R. norvegicus, harassment by the introduced Black Drongo Dicrurus macrocercus, and inbreeding depression (Morton et al. 1999, Plentovich et al. 2005, USFWS 2005, Wiewel et al. 2009, Sussman et al. in press). Habitat loss, fragmentation, and degradation may reduce nest success, especially given the Mariana Crow's specific nesting habitat requirements and limited availability of primary forests on Rota (Ha et al. 2011). Pesticides and pollutants have also been suggested as possible causes of the decline of the Mariana Crow (Amar et al. 2008), and are sources of habitat degradation which can reduce reproductive success (e.g. Fry 1995). Additionally, large typhoons in 1988 and 1997, which caused island-wide destruction of mature forest, may have played a role in reducing habitat quality (Morton et al. 1999). Lower reproductive success in isolated populations, such as those on islands, can also be attributed to deleterious effects of inbreeding (Rowley et al. 1993). Finally, while the brown tree snake is absent from Rota, other introduced predators may be a problem. Recent field evidence from recovered remains of Mariana Crows using radio telemetry suggests that feral cats are contributing to the Mariana Crow's population decline (S. Faegre and R. Ha, unpublished data).

Some aspects of Mariana Crow breeding ecology are well characterised. Morton et al. (1999) monitored the activities of 32 Mariana Crow pairs on Rota during the 1996-1998 breeding seasons. The Mariana Crow nesting period is approximately 74 days, with on average 15 days for nest building, 21.5 days for laying and incubating and 37.5 days for chick rearing (Morton et al. 1999). Both male and female participate in nest-building and biparental care is exhibited throughout all stages of offspring rearing. The majority of pairs $(67 \%)$ make more than one nesting attempt within a breeding season following a nesting failure, with a mean of 2.05 nesting attempts per pair per season ( $n=75$, Morton et al. 1999).

Unlike some other tropical and subtropical corvid species, Mariana Crows do not show signs of cooperative breeding and fledglings disperse from their natal territory at an average of 8 months (range: 3-18 months) after fledging and before the next breeding attempt by their parents (Morton et al. 1999). Mariana Crows usually nest in old-growth limestone forests, in areas with 
dense canopy cover and high densities of nesting materials such as woody vines (Ha et al. 2011). There is an average of one Mariana Crow pair per 22 ha of primary forest (Morton et al. 1999).

In this study, we compare the results of direct counts of territorial crow pairs on Rota during the 2007 breeding season with results of a similar count at the end of the 1998 breeding season (Plentovich et al. 2005). We examine the breeding performance of Mariana Crows from 1996 to 2009, and describe differences in nesting success related to location, time of year, human proximity, and period of study. We discuss reasons for these patterns in nesting success and population changes, and the avenues for recovery of this species.

\section{Methods}

\section{Study area}

Rota is the second most southerly island after Guam in the Mariana Islands, Western Micronesia $\left(14^{\circ} \mathrm{O} 9^{\prime} \mathrm{N}, 145^{\circ} 12^{\prime} \mathrm{E}\right)$. The $86-\mathrm{km}^{2}$ island is volcanic in origin with uplifted limestone terraces. The climate is tropical, with high humidity, and the wet season lasts from July to November. It is located within the Western Pacific typhoon belt, and experiences typhoons periodically. During the field years contributing to this study, Rota experienced several major typhoons, two of which occurred during the 1997 breeding season. Around $60 \%$ of the island is covered in native limestone forest (Falanruw et al. 1989), much of which is secondary growth (Engbring et al. 1986). The most recent significant clearing occurring in the mid-1990s, when a large forest area was cleared for the construction of the Rota Resort and golf course.

The majority of pair and nest monitoring in the 1996-1998 breeding seasons (see Mariana crow breeding biology and nesting success, below) was conducted in six primary study plots each approximately $\mathrm{I} \mathrm{km}^{2}$ (Figure $\mathrm{I}$ ). These plots continued to be sampled in subsequent years, but additional areas were added to provide more island-wide coverage, and because birds were

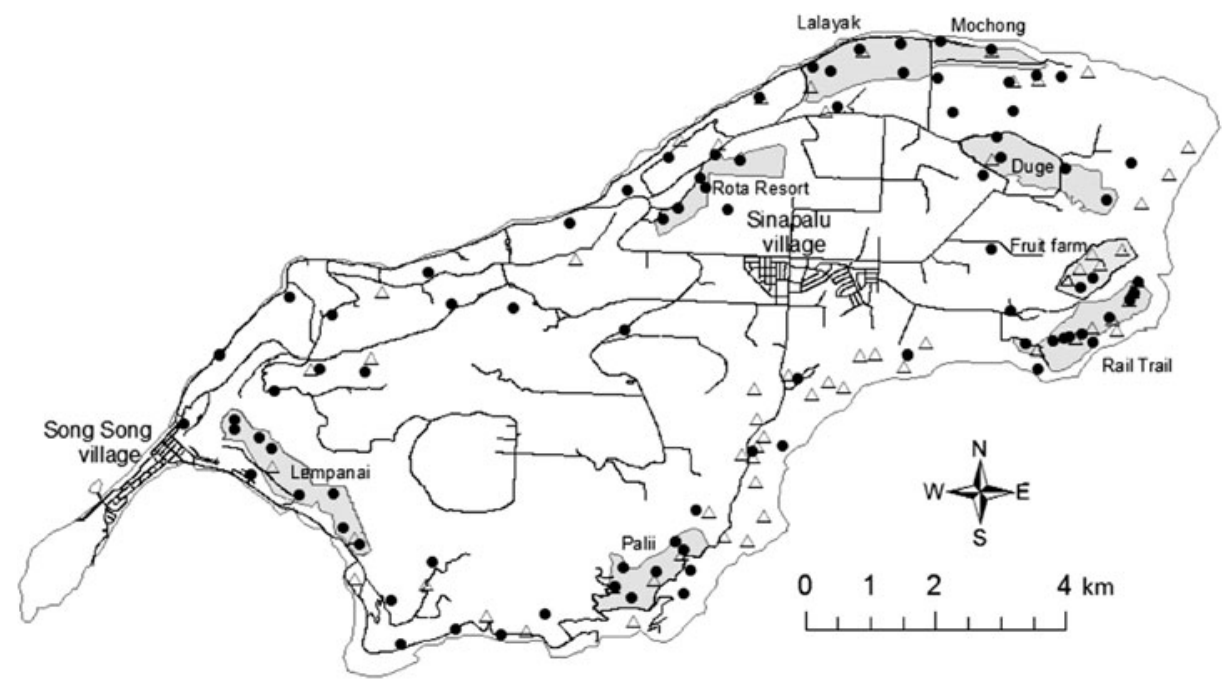

Figure 1. Map of Rota, with 85 known Mariana Crow pair locations from the 1998 survey marked with • (from Plentovich et al. 2005, excluding 32 extrapolated pairs) and 6o Mariana Crow pair locations from the 2007 survey marked with $\triangle$. The six study plots used for comparison of the change in Mariana Crow pair numbers from 1998 to 2007 (Duge, Rail Trail, Rota Resort, Mochong, Lalayak and Palii) and two newer study plots added in 1999 (Lempanai and Fruit Farm) are indicated. 
declining in some of the original study plots. These six study plots were originally selected because they were large contiguous land parcels that were being considered for habitat conservation, and were vegetated by forest habitat types representative of Rota (Morton et al. 1999, Ha et al. 2011).

\section{Mariana Crow population count}

We determined the current number of territorial Mariana Crow pairs present on Rota through intensive, island-wide searching and pair monitoring over an 8-month period from September 2007 to April 2008. We monitored the status and breeding attempts of all Mariana Crow pairs known from previous years (i.e. 1999-2006) and concurrently searched for new pairs in all areas of suitable habitat which had not yet been intensively searched and monitored. When possible, we confirmed new pairs by locating active nests or adults with fledglings, but in the absence of confirmed nests or fledglings, we used multiple Mariana Crow pair sightings (pair status determined by behaviours listed below) on different days within the same territory to confirm a territorial pair. In the absence of observations of a nest or fledglings, pairs were identified based on pair and territorial behaviour such as defensive calls, head banging, repeated bill swiping, stick throwing, pair preening and pair feeding. We combined the information from searches of new areas with our knowledge from the ongoing monitoring of known pairs, to estimate the total number of Mariana Crow pairs that occurred on Rota during the 2007 breeding season.

We compared this island-wide estimate of pairs observed in the 2007 breeding season with a previous island-wide estimate of 117 breeding pairs at the end of the 1998 breeding season (Plentovich et al. 2005). The Plentovich et al. (2005) estimate was based on cumulative knowledge of pairs from observations and monitoring during 1990-1999, and included 85 known breeding pairs both within and outside the six study plots, and an estimated 32 additional pairs based on extrapolating a density estimate (one pair per 22 ha) to other, unsearched areas of suitable habitat on Rota. We compared the total island-wide pair estimates in 1998 and 2007, as well as the number of pairs in the areas that were directly surveyed in both years (excluding the 32 extrapolated pairs from the 1998 pair estimate).

Uncertainty about the density extrapolation in the 1998 breeding season estimate prompted us to also compare pair losses within the six plots in which pairs were closely monitored for breeding activity. Comparisons were made from the 1998 season and the 2007 season as these breeding seasons were most similar in monitoring effort and prior monitoring history (and therefore prior knowledge of pair locations).

To evaluate the potential effects of human activities on Mariana Crow population changes, we assumed road length and type (paved versus unpaved) were reasonable indicators of human activities including disturbance (traffic volume and related noise) and access (sight-seeing by tourists, subsistence use by residents). We measured the length of paved and unpaved roads with and directly adjacent to each study plot using Quickbird satellite imagery from 2002. We used Spearman's rank correlation to test the association between the retention of breeding pairs on study plots with the ratio of study area to road length for each study plot. Paved roads were doubled in length to account for higher levels of traffic volume and associated ongoing human disturbances relative to unpaved roads. Given the small sample size of the analysis $(n=6)$, we intended this analysis to be mainly descriptive.

\section{Mariana Crow breeding biology and nesting success}

We considered the Mariana Crow breeding season to extend from I July to 30 June of the following year. For example, the 2009 breeding season covered the period July 2009-June 2010. We assigned nests to an individual season using the calendar year in which the season began (i.e. a clutch that was initiated on I January 2005 was included in the 2004 breeding season). We monitored known territorial crow pairs for signs of nesting in each breeding season from 1996 to 2009 , 
except for the 2002 and 2004 seasons when there were no researchers monitoring crows on Rota. From 1996 to 1998 six study plots were monitored (Figure 1): Duge, Lalayak, Mochong, Palii, Rail Trail, and the Rota Resort. In 1999 two new areas were added: Lempanai and Fruit Farm. The months in which monitoring took place varied among years but, as a minimum, monitoring always occurred from September/October to March/April each year.

When a nest was found, nest status (i.e. complete, laying, egg, or nestling) was determined approximately once a week by observing the nest from the ground. Nests were checked more frequently around expected fledging dates. The contents of the nests were generally checked with a mirror pole (where possible) once during incubation and once during the nestling stage. When mirror poles were not used, the presence of eggs or nestlings was determined based on behavioural observations of adults or direct observations of nestlings. Nests were only considered "active" (and included in data analysis) if they contained eggs or nestlings. Some nests were videotaped (in 1997 and 2007-2009) during the incubation and nestling periods. Clutch initiation date was defined as the date that the first egg was laid. Since we rarely knew this lay date from direct observation, the date was interpolated by calculating the midpoint between observed periods of incubation, nestling and fledgling to calculate key dates of laying, hatching and fledging, and then counting back from the most accurately known key date given known clutch size/incubation period/nestling periods. We used the average laying duration of 1.6 days, incubation period duration of 21.5 days, and nestling period duration of 37.5 days. During the 1999, 2000, 2001 and 2003 breeding seasons, eggs or nestlings from some nests were removed and transported to Guam for captive rearing and release on Guam.

We calculated nest success rates using the nest survival option in Program MARK, following the methods described by Dinsmore et al. (2002), Jehle et al. (2004) and Rotella et al. (2004). As not all pairs were banded, and many were not tracked between breeding seasons, we were unable to study nest success as a repeated measure within pairs over field seasons. We included time of season (number of days since the first day of the nest season), nest age (number of days since clutch initiation) and breeding season as independent predictor variables on daily survival rates of nests. We used AICc to evaluate models, and chose models with the lowest AICc or those within two units to be supported in explaining the variation in the data.

We also examined nest success as defined by the proportion of pairs that successfully produce at least one fledgling per breeding season. We calculated the Pearson correlation coefficient to determine if there was a linear trend in pair productivity by year. Statistical significance was set at $\alpha=0.05$ for all analyses.

\section{Results}

\section{Mariana Crow population count}

We identified 6o pairs of crows across the island during September 2007 to April 2008 (Figure 1), as well as 24 unpaired birds, including a flock of 14 in the Fruit Farm region. The rate at which we found new crow pairs reached an asymptote in late January (Figure 2), despite continued search effort, indicating that our count was not limited by time spent searching.

Plentovich et al. (2005) estimated 117 pairs of Mariana Crows island-wide by the end of the 1998 breeding season. This estimate included 31 Mariana Crow pairs monitored in the six study plots, 54 pairs identified elsewhere on Rota, and 32 pairs that were assumed based on density extrapolations to areas that were not surveyed but contained suitable habitat. Our 2007 pair estimate of 60 represents at least a $30 \%$ decline in pair numbers (based on 85 pairs that were directly counted in 1998), but more probably a $49 \%$ decline (based on 117 pairs, including both pairs that were directly counted and pairs that were extrapolated in the first survey), between 1998 and 2007. The number of Mariana Crows found within the six study plots (see Methods) also declined, from 28 pairs in the 1998 breeding season to only 15 pairs 


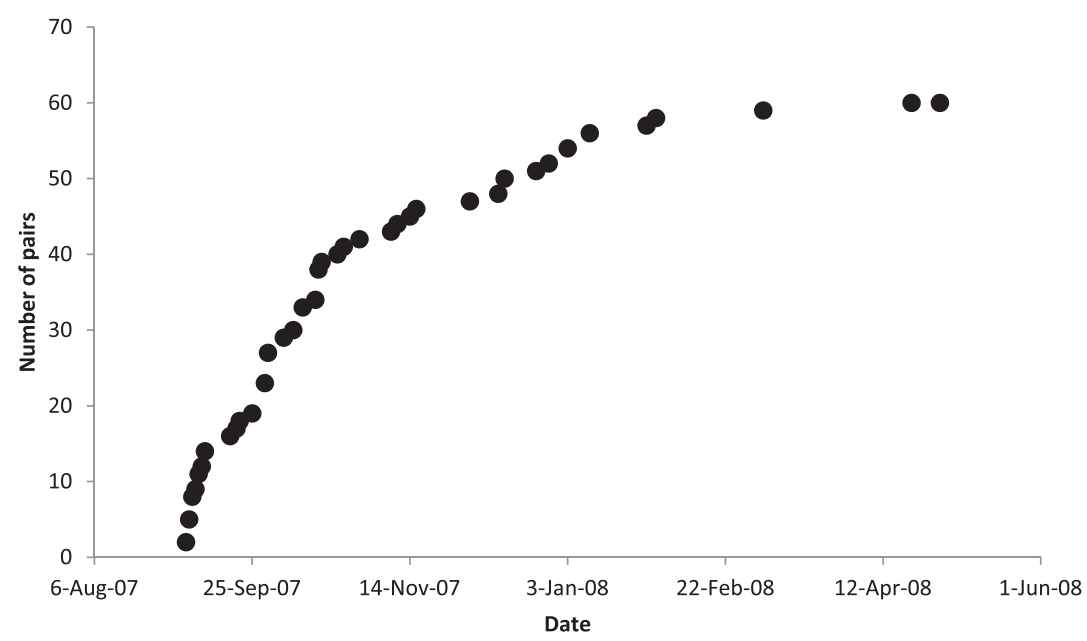

Figure 2. Cumulative number of Mariana Crow territorial pairs identified on Rota during 2007 breeding season in relation to date. Search effort (through quarterly road station surveys, study plot searching and nest monitoring, and searches in previously unsearched areas) was consistent throughout.

in the 2007 breeding season, a loss of $46 \%$ in 9 years. By 2009 the loss was even greater, with only 11 pairs found in 2008 and 2009, representing a 61\% decline in the study plots since 1998 (Table 1). Retention of pairs appeared greater in plots such as Palii and Rail Trail that were furthest from villages and roads (Table 1, Figure I). A Spearman's rank correlation suggested that there was a relationship between number of pairs and potential disturbance (ratio of study area to road length within/adjacent to plot) that trended towards significance in this small sample (rho $=0.77, P=0.10, n=6)$.

\section{Mariana Crow breeding biology and nesting success}

We found and monitored 204 active Mariana Crow nests on Rota from the 1996 to 2009 breeding seasons. There was an average of 2.57 eggs per nest ( $\mathrm{SD}=0.8, n=82), 1.39$ nestlings per nests in nests that hatched $(S D=0.5, n=106)$, and 1.25 fledglings per nest in nests that

Table 1. Number of territorial pairs located during breeding seasons in each of six study plots on Rota during 1996-2009 breeding seasons. Column values are number of pairs confirmed present in each study plot in each breeding season.

\begin{tabular}{lcclcccc}
\hline & \multicolumn{2}{l}{ Study plot } & & & All \\
\cline { 2 - 7 } Breeding season & Duge & Lalayak & Mochong & Palii & Rail Trail & Resort & \\
\hline 1996 & 3 & 5 & 3 & 5 & 8 & 6 & 30 \\
1997 & 3 & 5 & 3 & 4 & 8 & 5 & 28 \\
1998 & 4 & 5 & 3 & 4 & 8 & 4 & 28 \\
2007 & 1 & 2 & 1 & 3 & 7 & 1 & 15 \\
2008 & 0 & 0 & 1 & 3 & 6 & 1 & 11 \\
2009 & 0 & 0 & 1 & 3 & 6 & 1 & 11 \\
$\%$ 1998 pairs in 2007 & 25 & 40 & 33 & 60 & 88 & 25 & 54 \\
\% 1998 pairs in 2009 & 0 & 0 & 33 & 60 & 75 & 25 & 39 \\
\hline
\end{tabular}


fledged ( $\mathrm{SD}=0.4, n=68)$. Using the nest survival estimation in Program MARK, the overall daily survival rate for nests was 0.978 (SE 0.00216), giving an overall nest success rate of $25.7 \%$ for an average 60.6 day nest cycle. Models suggested that nests were more likely to survive later in the nest cycle. There was also some support from one of the two best models that nest survival rates declined in more recent breeding seasons (Table 2). However, nest start date (i.e. timing within the season) did not appear to affect the survival rate of nests (Table 2).

\section{Breeding success by pair}

An alternative measure of success is the proportion of monitored pairs that produced at least one fledgling per breeding season. This varied from a low of 0.21 in the 1997 season (during which several typhoons caused many nest failures) to a high in the following season (1998) of 0.73 , with an overall rate of 0.49 over the entire study period, or 0.52 if the 1997 season is omitted (Table 3). The rate of breeding success per pair per season did not show an overall linear trend (Pearson correlation coefficient $=0.120, P>0.05, n=8)$. The number of fledglings per pair per year ranged from 0.21 in 1997 to 1.095 in 1998 , with an average of 0.66 fledgling per pair per year (Table 3). The number of fledglings per pair per year also showed no overall linear trend (Pearson correlation coefficient $=-0.00851, P>0.05, n=8$ ).

\section{Causes of Mariana Crow nest failures}

Of the 204 nests monitored, 70 were successful in producing at least one fledgling, 105 failed, 12 were harvested for the Guam translocation program, and 17 had unknown outcomes (Table 4). The 105 failed nests included 39 that appeared depredated, nine destroyed by typhoons, five in which chicks were found ill or dead, three which failed to hatch, two abandoned due to human interference, and 47 that failed for undetermined reasons. The two nests that were abandoned due to human interference were a result of filming attempts that caused abandonment due to the camera being too close in 1997-later filming attempts in 2007 onwards were carried out with cameras placed at least $20 \mathrm{~m}$ from the nests so that they did not cause abandonment. Of the depredated nests, three episodes were captured on film. Two nests were depredated by Mariana Crows, one during the incubation stage and one during the nestling stage, and one nest was depredated by Micronesian Starlings Aplonis opaca during the incubation stage. Additionally, one clutch may have been taken by a monitor lizard, based on the large hole in the depredated nest (Morton et al. 1999). Four of the nest failures in which nestlings were found ill or dead occurred in the Rota Resort plot during the 1997-1999 breeding seasons; a fifth was found in the Rail Trail plot during the 2009 season. As many nest predation events do not leave evidence, the 48 nests for which the cause of failure was undetermined probably include a high percentage of predation events. Thus it is highly likely

Table 2. Daily survival rate maximum likelihood estimation using Program MARK Nest Survival. Nest age $=$ number of days since first egg laid. Linear date $=$ number of days since first day of season that the first egg was laid. Year = breeding season. $\%$

\begin{tabular}{lllll}
\hline Model & AICc & $\triangle$ AICc & Weight & Parameters \\
\hline Nest age + year & 592.1464 & 0.0000 & 0.46320 & 2 \\
Nest age & 592.5754 & 0.4290 & 0.37377 & 2 \\
Nest age + linear date & 594.3091 & 2.1627 & 0.15709 & 3 \\
Year & 602.8241 & 10.6777 & 0.00222 & 1 \\
Linear date & 602.8586 & 10.7122 & 0.00219 & 2 \\
Constant DSR & 603.5696 & 11.4232 & 0.00153 & 1 \\
\hline
\end{tabular}


Table 3. Mariana Crow pair success and productivity during 1996-2009 breeding seasons. Table shows proportion of monitored pairs that produced at least I fledgling ("Success/pair") and the number of fledglings per pair per season ("Productivity/pair"). Seasons when translocations occurred prior to fledging or when there were too few pairs monitored (less than 10) for a reliable estimate of productivity are indicated. Two major typhoons in 1997 significantly affected nesting outcomes that year, so we also include totals without the 1997 data.

\begin{tabular}{lllll}
\hline Season & Number of Pairs & $\begin{array}{l}\text { Number with } \\
\text { fledglings }\end{array}$ & Success/pair & $\begin{array}{l}\text { Productivity/ } \\
\text { pair }\end{array}$ \\
\hline 1996 & 10 & 0.63 & 0.76 \\
1997 & 16 & 3 & 0.21 & 0.21 \\
1998 & 14 & 16 & 0.73 & 1.095 \\
1999 & 22 & 1 & No data & No data \\
2000 & Translocations & & Too few data & Too few data \\
2001 & 5 & 4 & No data & No data \\
2003 & Translocations & & Too few data & Too few data \\
2005 & 9 & 4 & Too few data & Too few data \\
2006 & 4 & 4 & 0.36 & 0.36 \\
2007 & 11 & 9 & 0.60 & 0.70 \\
2008 & 15 & 6 & 0.60 & 0.713 \\
2009 & 10 & 9 & 0.60 & 0.75 \\
Average & 15 & & 0.49 & 0.66 \\
Average w/out 1997 & & & 0.52 & 0.72 \\
\hline
\end{tabular}

that we have underestimated the prevalence of nest predation as a cause of nest failure in Mariana Crows. A summary of nest outcomes by plot is shown in Table 4.

\section{Mariana Crow renesting attempts}

Overall, we found that $44 \%$ of pairs that failed on their first nest made at least one renesting attempt. However, this figure could have been affected by the different monitoring methods used in some later seasons when the priority was to monitor a large number of pairs for the

Table 4. Outcomes of 204 Mariana Crow nests during 1996-2009 breeding seasons, determined by nest checks and nest filming, by study plot. "Other" means that the nest was located outside existing study plots. "Undetermined failure" means the cause of failure was not determined, "unknown outcome" means the outcome of the nest (success or failure) was unknown.

\begin{tabular}{|c|c|c|c|c|c|c|c|c|c|c|}
\hline \multirow[b]{2}{*}{ Outcome } & \multicolumn{9}{|c|}{ Study plot } & \multirow{2}{*}{$\begin{array}{l}\text { Total (\% of } \\
\text { all failures) }\end{array}$} \\
\hline & Duge & Lala. & Moch. & Palii & Rail T. & Resort & Fruit F. & Lemp. & Other & \\
\hline Predation & 2 & 4 & 6 & 9 & 11 & 5 & 2 & o & o & $39(37 \%)$ \\
\hline Typhoon & o & 2 & 4 & 2 & 1 & o & o & o & o & $9(8.5 \%)$ \\
\hline Nstl. ill/dead & o & o & o & o & 1 & 4 & o & o & o & $5(5 \%)$ \\
\hline Infertile & o & 2 & o & 1 & o & o & o & o & o & $3(3 \%)$ \\
\hline Human cause & 1 & 1 & o & o & o & o & o & o & o & $2(2 \%)$ \\
\hline $\begin{array}{l}\text { Undetermined } \\
\text { failures }\end{array}$ & 1 & 1 & 8 & 9 & 9 & 2 & 8 & 6 & 3 & $47(45 \%)$ \\
\hline Translocated & 2 & o & 1 & o & 4 & $\mathrm{o}$ & 1 & 1 & 3 & 12 \\
\hline Success & 3 & 6 & 9 & 16 & 15 & 3 & 11 & 5 & 2 & 70 \\
\hline $\begin{array}{l}\text { Unknown } \\
\text { outcome }\end{array}$ & 1 & o & 4 & 7 & 2 & 1 & 1 & 1 & o & 17 \\
\hline Total & 10 & 16 & 32 & 44 & 43 & 15 & 23 & 13 & 8 & \\
\hline
\end{tabular}


purpose of the translocation program. During these seasons, many individual pairs were not monitored frequently enough to quantify all renesting attempts. Consequently, we expect that the actual rate of renesting after a failure was significantly higher than this figure. Many pairs made up to three nest attempts per season. The majority of renesting attempts (93\%) occurred after previous nests failed, although three renesting attempts followed a successful fledging in the same season. Of these attempts, two pairs were able to produce two successful nests within a single season (i.e. double brooding); the third pair was not successful in their second nesting attempt.

\section{Discussion}

We counted 6o territorial Mariana Crow pairs during the 2007 breeding season, a $49 \%$ decline since the last direct count in 1998. A similar sized decline (46\%) was found when comparing only the number of pairs within the six study plots. The declines in breeding pair numbers appear to be non-uniform across the island. The two plots with the lowest amount of pair loss (Palii and Rail Trail) had fewer roads and were relatively less accessible than the other study plots due to steep cliffs and few to no access roads (Table 1 , Figure 1 ). The relationship between amount of roads in a plot and pair loss trended towards significance, though given the small sample size and low power, the analysis was not conclusive. More direct measures of human activities are needed to evaluate this possible relationship, such as comparison of changes in forest cover or forest edge:area ratio with crow pair losses. It suggests the possibility of a negative impact of human disturbance on the Mariana Crow population on Rota, possibly due to habitat degradation and fragmentation, human persecution, or an increase in predators such as feral cats, which may be more numerous in areas closer to human habitation. In support of this idea, the higher retention of pairs in the Rail Trail study plot may have been the result of cat control efforts at this site during 1997 and 1998, linked to the introduction of the Guam Rail Gallirallus owstoni. There is also evidence for direct human persecution. In 1995, two dead adult Mariana Crows were found below their nest with bullet wounds (USFWS 2005). A recent survey suggested that these might not be isolated incidents, as some Rota residents condoned behaviour such as chasing and shooting Mariana Crows (Sussman et al. in press).

There have been several Mariana Crows (both adults and juveniles) seen with crossed bills or foot deformities such as feet permanently clenched in a ball, extra-long toes and/or toenails, missing toes, and toes sticking at odd angles so that the individual cannot grip a branch. Twice such deformities in a juvenile have resulted in that juvenile being dependent on the adults for over a year, and preventing those adults from attempting to breed in the following breeding season (J. Morton pers. comm. in Hunt et al. 2012, L. Zarones pers. obs.). These incidences were not always deleterious or permanent-one individual with a crossed bill was able to successfully breed as an adult and was later observed with a normal-looking bill (S. Faegre pers. obs.). We do not know the cause of these deformities: whether disease, injury or inbreeding. Genetic inbreeding of a declining population with a founder effect (Tarr and Fleischer 1999) may push an already stressed population to further decline and eventual extinction.

The impact of inbreeding on Mariana Crow nest success needs to be explored in greater detail. Past research on island avifauna has shown that genetic bottlenecks within populations can dramatically decrease hatchability (Briskie and Mackintosh 2004) and that inbred populations show depressed reproductive fitness compared with closely related, outbred species (Frankham 2005). Briskie and Mackintosh's (2004) review of studies of New Zealand species suggested that a bottleneck of about 150 birds, or 75 pairs, appeared to be a critical threshold, under which hatchability decreased rapidly. Our 2007 count of just 6o pairs is therefore worrying, as it suggests that Mariana Crows on Rota could be at risk for detrimental effects of inbreeding. The risk of inbreeding due to small population size is compounded by the fact that the Rota population has already shown reduced genetic diversity even before the continued decline documented in this study. Tarr and Fleischer (1999) found that the Rota population was 
more genetically similar than the now extirpated Guam population, even though the Guam population during the 1990s at the time of the study was smaller and less stable. Several studies have shown that inbreeding can result in increased physiological or behavioural abnormalities (e.g. Crnokrak and Roff 1999). We have observed abnormal nest building behaviour among Mariana Crows on several occasions, such as the excessive deposition of sticks at the nest site, resulting in an accumulation of nesting material on the ground below nests to a height of nearly i m (Morton et al. 1999). Several such nests were added to for a period of several months but never completed (S. Faegre pers. obs.). These behaviours might indicate inbreeding or simply inexperience in nest building.

\section{Mariana Crow nesting success}

Although annual nest success and pair success varied among years, there was no overall trend over time. From the causes of nest failures identified, the most common cause was predation, although from our data we could not conclusively identify which predators were most important for eggs or nestlings. Nest filming showed crows and starlings consuming the nest contents, although the sample size was small. Another study has revealed that high abundance of introduced rats around Mariana Crow nests did not decrease breeding success, suggesting that rats may not be important nest predators for this species (Amar and Esselstyn 2013.). Indeed Amar and Esselstyn (2013) found a significant positive relationship between rat abundance and Mariana Crow breeding success; it was proposed that this relationship may be due to the abundance of a shared predator, the feral cat. Predation by feral cats has also been proposed for the widespread declines of other bird species on Rota (Amar et al. 2008). There is evidence that feral cats are predators of adult and juvenile Mariana Crows (S. Faegre and R. Ha unpubl. data from nine radio-tracked birds and one un-banded bird) and could also be a potential threat to nests. Large nestlings have been taken from several nests, suggesting predation either by cats or large monitor lizards. One attempted predation of a large nestling by a feral cat was observed, although the parents successfully drove the cat away before it reached the nest (S. Faegre pers. obs.). Furthermore, the fate of the Mariana Crow on Guam is an illustration of the devastating effects introduced predators can have on island species (Savidge 1987, Wiles et al. 2003).

Another factor affecting nesting success was the impact of typhoons. Our data included observations for one year when Rota experienced a major typhoon, 1997. Presumably due to the effects of the storm, we recorded the lowest success per pair and productivity per pair of any study year during 1997 (see Table 3). Simulations of global climate change predict an increase in the intensity of tropical cyclones and typhoons (Meehl et al. 2007). If crow populations continue to decrease, the impact of such storms may become even more devastating for the species.

The Mariana Crow's proclivity for renesting is a characteristic that may be advantageous in their conservation. Mariana Crows were capable of successfully renesting during a breeding season after a failed nest attempt, with subsequent clutches initiated on average about 40 days after the previous nesting attempt failed. Overall, $44 \%$ of pairs that failed on their first attempt renested at least once. However, since not all pairs were monitored intensively during some years, this percentage may be biased low-we believe that the Morton et al. (1999) estimate of $67 \%$ may be a more accurate measure of renesting rates, due to more intense monitoring of specific pairs during the 1996-1998 breeding seasons than in later years. On two occasions, pairs were observed to successfully fledge offspring from two nests within the same season. Both pairs successfully reared two sets of offspring through to dispersal and then nested again at the beginning of the subsequent breeding season (Morton et al. 1999, S. Faegre and R. Ha unpubl. data).

The daily survival rate of nests decreased during the study period. This contrasts with the proportion of pairs with at least one successful nest per year, and the average number of fledglings per pair per year, which did not decrease during the study period. The ability of Mariana Crows to renest after failure combined with the 9-month breeding season may mean that decreasing daily survival rates (and ultimately nest success rates) can be mitigated by renesting and eventual success. 
The Hawaiian Crow Corvus hawaiiensis, now extinct in the wild, is the only other tropical island crow for which nest success data are available. Estimates for clutch size between the Mariana Crow and the Hawaiian Crow are fairly similar, with the Hawaiian Crow laying an average of 2.8 eggs per nest (Banko et al. 2002), compared with 2.57 for the Mariana Crow. The estimates for number of fledglings per successful nest are also similar, with Hawaiian Crows fledging an average of 1.32 chicks as opposed to the 1.25 chicks per successful nest for the Mariana Crow. However, a comparison of the average number of fledglings per pair per year suggests that Mariana Crows are less productive than Hawaiian Crows. Sixty-three percent of Hawaiian Crow pairs and $49 \%$ of Mariana Crow pairs succeeded in producing at least one chick per year. The declining population of Hawaiian Crows fledged an average of $0.72-0.86$ young per pair each year (Jenkins 1983, Giffen et al. 1987) whereas the declining population of Mariana Crows fledged an average of 0.66 fledglings per pair per year between 1996 and 2009. These results suggest that although clutch sizes and number of fledglings per pair are similar, the overall measures of reproductive output are higher for Hawaiian Crows than for Mariana Crows. This is despite the relatively short breeding season of four months for the Hawaiian Crow (Banko et al. 2003), compared with nine months for the Mariana Crow.

\section{Conclusions}

The number of crow territorial pairs has declined by around 50\% on Rota from 1998 to 2007 . The association between road density and pair retention suggests that human activities could be a factor in the declines, but more direct measures of disturbance are needed. Predation appears to be a major cause of nest failure in this species. Mariana Crows have an extended breeding season (up to nine months), and are capable of renesting up to three times in a breeding season following nest failure, with similar breeding success rates between first and subsequent nests. Despite this, the annual reproductive rate of pairs is low, compared to the Hawaiian Crow, and the cause of this is unknown. Feral cats are known to be a cause of mortality for both adults and juveniles. We strongly recommend a combined program of feral cat control, habitat protection, public outreach and population augmentation through captive rearing and/or surrogate parenting to ensure the continued survival of the Mariana Crow.

\section{Acknowledgements}

We are grateful for the critical reviews of earlier versions of this manuscript by anonymous reviewers. This study was only possible because of the excellent professionalism shown by numerous biologists over the years: F. Amidon, M. Awasthy, M. Colon, G. Desy, B. Dicke, R. Dymzarov, J. Esselstyn, S. Farry, J. Graham, P. Hannon, J. Herriges, S. Holman, B. Hughes, J. Jackson, N. Johnson, M. Knight, S. Lawson, T. Lee, K. Levenstein, K. Livengood, B. Massey, B. O'Neil, M. Parker, M. Philippart, S. Pruett, L. Pulliam, J. Quitugua, R. Reed, E. Rehm, T. Reid, G. Robinson, D. Rollinson, M. Rogne, S. Seibel, T. Sharp, J. Shousky, N. Shutt, J. Stanaway, L. Tanino, R. Ulloa, M. Vamstad, E. Weiser, E. Wells, C. Willet, S. Wyshynski, and additional staff over the years from Guam's Division of Aquatic and Wildlife Resources. L. Williams and S. Kremer provided logistical support, and S. Taisacan and D. Worthington provided field support, for work on Rota CNMI. We thank the many CNMI Department of Lands and Natural Resources Secretaries and Division of Fish and Wildlife Directors who have supported this work over the years, including A. Palacios, M. Pangelinan, I. Dela Cruz, S. Igisomar, R. Seman, and P. Hamilton. All research was conducted under Federal Endangered Species Permits FWSPIO-3, TE-032209-7/8 and TE-017352-14 Federal Bird Banding Permits 22570 and 22802, CNMI scientific collecting permit no. 01512, and an amended CNMI Fish and Game Permit o68-95-SPN. This work was financially supported by the Commonwealth of the Northern Mariana Islands, Division of Fish and Wildlife and the U. S. Fish \& Wildlife Service (Endangered Species, Section 6). 


\section{References}

Aguon, C. F., Campbell, E. W. and Morton, J. M. (2002) Efficacy of electrical barriers used to protect Mariana crow nests. Wildl. Soc. Bull. 30: 703-708.

Amar, A. and Esselstyn, J. A. (2014) Positive association between rat abundance and breeding success of the critically endangered Mariana crow Corvus kubaryi. Bird Conserv. Internatn. 24: 192-200.

Amar, A., Amidon, F., Arroyo, B., Esselstyn, J. A. and Marshall, A. P. (2008) Population trends of the forest bird community on the Pacific island of Rota, Mariana Islands. Condor I10: $421-427$.

Banko, P. C., Bell, D. L. and Banko, W. E. (2002) Hawaiian crow: Corvus hawaiiensis. In A. Poole and F. Gill, eds. Birds of North America, 648. Philadelphia, PA USA: The Academy of Natural Sciences, and Washington DC, USA: The American Ornithologists' Union.

Briskie, J. V. and Mackintosh, M. (2004) Hatching failure increases with severity of population bottlenecks in birds. Proc. Nat. Acad. Sci. 101: 558-561.

Camp, R. J., Pratt, T. K., Marshall, A. P., Amidon, F. and Williams, L. L. (2009) Status and trends of the land bird avifauna on Saipan, Mariana Islands, with emphasis on the endangered Nightingale Reed-Warbler (Acrocephalus luscinia). Bird Conserv. Internatn. 19: 323-337.

Camp, R. J., Amidon, F. A., Marshall, A. P. and Pratt, T. K. (2012) Bird populations on the island of Tinian: persistence despite wholesale loss of native forests. Pac. Sci. 66: 283-298.

Crnokrak, P. and Roff, D. A. (1999) Inbreeding depression in the wild. Heredity 83: 260-270.

Dinsmore, S. J., White, G. C. and Knopf, E. L. (2002) Advanced techniques for modeling avian nest survival. Ecology 83: 3476-3488.

Engbring, J, Ramsey, F. L. and Wildman, V. J. (1986) Micronesian forest bird survey, 1982: Saipan, Tinian, Aguiguan, and Rota. Washington, D.C. USA: U.S. Fish \& Wildlife Service, Department of the Interior.

Falanruw, M. C., Cole, T. G. and Ambacher, A. H. (1989) Vegetation survey of Rota, Tinian, and Saipan, Commonwealth of the Northern
Mariana Islands. Berkeley, California USA: Pacific Southwest Forest and Range Experiment Station.

Fancy, S. G., Lusk, M. R. and Grout, D. (1999) Status of the Mariana crow population on Rota, Mariana Islands. Micronesica 32: 3-10.

Frankham, R. (2005) Genetics and extinction. Biol. Conserv. 126: 131-140.

Fry, D. M. (1995) Reproductive effects in birds exposed to pesticides and industrial chemicals. Environ. Health Persp. 103: 165-171.

Giffen, J. G., Scott, J. M. and Mountainspring, S. (1987) Habitat selection and management of the Hawaiian crow. J. Wildl. Manage. 51: 485-494.

Ha, R. R, Morton, J. M., Ha, J. C., Berry, L. and Plentovich, S. (2011) Nest site selection and consequences for reproductive success of the endangered Mariana crow (Corvus kubaryi). Wilson J. Ornithol. 123: 236-242.

Hunt, G. R., Holzhaider, J. C. and Gray, R. D. (2012) Prolonged parental feeding in toolusing New Caledonian Crows. Ethology 118: $1-8$.

IUCN (2011) IUCN Red List of threatened species. <http://www.iucnredlist.org/apps/ redlist/details/106005766/o> (7 February 2012).

Jehle, G., Yackel Adams, A. A., Savidge, J. A. and Skagen, S. K. (2004) Nest survival estimation: A review of alternatives to the Mayfield estimator. Condor 106: 472-484.

Jenkins, J. M. (1983) The native forest birds of Guam. Ornithol. Monogr. 31.

Meehl, G. A., Stocker, T. F., Collins, W. D., Friedlingstein, P., Gaye, A. T., Gregory, J. M., Kitoh, A., Knutti, R., Murphy, J. M., Noda, A., Raper, S. C. B., Watterson, I. G., Weaver, A. J. and Zhao, Z. C. (2007) Global climate projections. In S. Solomon et al. eds. Climate change 2007: The physical science basis. Contribution of Working Group I to the Fourth Assessment Report of the Intergovernmental Panel on Climate Change. Cambridge, UK and New York, NY: Cambridge University Press.

Michael, G. A. (1987) Notes on the breeding biology and ecology of the Mariana or Guam crow. Avicultural Mag. 93: 73-82. 
Morton, J. M., Plentovich, S. and Sharp, T. (1999) Reproduction and juvenile dispersal of Mariana crows (Corvus kubaryi) on Rota, 1996-1999. Honolulu, HI. USA: U.S. Fish \& Wildlife Service, Pacific Islands Ecoregion.

Newton, I. (1998) Population limitation in birds. San Diego, California USA: Academic Press. Plentovich, S., Morton, J. M., Bart, J., Camp, J. R, Lusk, M., Johnson, N. and Vanderwerf, E. (2005) Population trends of Mariana Crow Corvus kubaryi on Rota, Commonwealth of the Northern Mariana Islands. Bird Conserv. Internatn. 15: 211-224.

Rodda, G. H. and Savidge, J. A. (2007) Biology and impacts of Pacific Island invasive species. 2. Boiga irregularis, the Brown Tree Snake (Reptilia: Colubridae). Pac. Sci. 61: 307-324. Rotella, J., Dinsmore, S. J. and Shaffer, T. L. (2004) Modelling nest-survival data: a comparison of recently developed methods that can be implemented in MARK and SAS. Anim. Biodiv. Conserv. 27: 187-205.

Rowley, I., Russell, E. and Brooker, M. (1993) Inbreeding in birds, Pp. 304-328 in N. W. Thornhill, ed. The natural history of inbreeding and outbreeding: theoretical and empirical perspectives. Chicago, California USA: University of Chicago Press

Savidge, J. A. (1987) Extinction of an island avifauna by an introduced snake. Ecology 68: 66o-668.
Steadman, D. (2006) Extinction and biogeography of tropical island birds. Chicago, USA: University of Chicago Press.

Sussman, A. F., Ha, R. R. and Henry, H. (in press) Evaluating the attitudes, knowledge, and practices affecting endangered birds and the potential for a landowners incentive program on Rota, CNMI. Oryx.

Tarr, C. L. and Fleischer, R. C. (1999) Population boundaries and genetic diversity in the endangered Mariana crow (Corvus kubaryi). Mol. Ecol. 8: 941-949.

USFWS (1984) Endangered and threatened wildlife and plants: determination of endangered status of seven birds and two bats of Guam and the Northern Mariana Islands. Federal Register 49: 33881-33885.

USFWS (2005) Draft revised recovery plan for the Aga or Mariana Crow, Corvus kubaryi. Portland, Oregon USA: U.S. Fish \& Wildlife Service.

Wiewel, A. S., Yackel Adams, A. A., Rodda, G. H. (2009) Distribution, density, and biomass of introduced small mammals in the southern Mariana Islands. Pac. Sci. 9: 205-222.

Wiles, G. J., Bart, J., Beck, R. E., Jr, and Aguon, C. F. (2003) Impacts of the brown tree snake: patterns of decline and species persistence in Guam's avifauna. Conserv. Biol. 17:1350-1360.

LAINIE ZARONES*1, ADRIENNE SUSSMAN, SARAH FAEGRE, RENEE ROBINETTE HA

Department of Psychology, University of Washington, 119A Guthrie Hall, UW Box 351525, Seattle, WA 98195, USA.

${ }^{1}$ Present address: Department of Lands and Natural Resources, Lower Base, PO Box 10007, Saipan, MP 96950 Northern Mariana Islands, Saipan, MP 96950, USA.

JOHN M. MORTON

U.S. Fish \& Wildlife Service, Pacific Islands Ecoregion, PO Box 8134, MOU-3 Dededo, GU 96912, USA.

Present address: U.S. Fish \& Wildlife Service, Kenai National Wildlife Refuge, P.O. Box 2139, Soldotna, AK 99669, USA.

\section{SHELDON PLENTOVICH}

Department of Zoology, Edmondson Hall 152, 2538 McCarthy Mall, University of Hawaii, Honolulu, HI 96822, USA. 
Present address: U.S. Fish \& Wildlife Service, Pacific Islands Coastal Program, 300 Ala Moana Blvd, Honolulu, HI 96813, USA.

\section{CELESTINO AGUON}

Division of Aquatic and Wildlife Resources, Department of Agriculture, 163 Dairy Road, Mangilao, GU 96913, USA.

\section{ARJUN AMAR}

CNMI-Divison of Fish and Wildife, P.O. Box 1397, Rota, MP 96951, USA.

Present address: Percy FitzPatrick Institute, DST/NRF Centre of Excellence, University of Cape Town, Rondebosch 7701, Cape Town, South Africa.

*Author for correspondence; email: lainiezarones@gmail.com

Received 23 July 2013; revision accepted 3 March 2014; Published online 8 October 2014 